\title{
THE ROLE OF RELAXIN HORMONE IN INTERNAL DERANGEMENT OF TEMPROMANDIBULAR JOINT
}

\author{
Ahmed Kamal Ahmed Shahin, Eman Abd El Halim EL Sharrawy * , Alaa El-Din Saad Abd El \\ Hamid ** , Tamer Abd El Bari Hamed *** \\ Professor of general anasethesiology Faculty of Dentistry Suez Canal University \\ ** Professor of Clinical Pathology Faculty of Medicine Suez Canal University \\ ***Assistant Professor of Oral and Maxillofacial Surgery Faculty of Dentistry Suez Canal University
}

\begin{abstract}
To assess the level of relaxin -2 hormone in the serum and synovial fluid in female patient who have anterior disc displacement and detect the effect the wash and lavage on it's level.Forty patients were included with age ranged between 20-40 age, old selected from outpatient clinic of oral and maxillofacial department faculty of dentistry, suez canal university. Patients were devided into two groups : group I(control group included 20 patients with normal tmj and group II included 20 patients with tmj internal derangement for patients preoperative assessment was done including standard pain scoring, maximal mouth opening and radiological examination also arthrocentesis with collecting of synovial fluid was performed serum relaxin -2 hormone \& synovial fluid relaxin -2 hormone were estimated using Elisa. results showed a significant increase in serum relaxin -2 hormone level in group II compared to control .this level was significantly decrease after 1 week after arthrocentesis. As regard to synovial fluid relaxin 2 hormone level,there was insignificant decrement in the hormone level after arthrocentesis . Clinical evaluation for the studied patients showed a high significant decreased in pain score after one week ,one month and at six months post-operative while, there was a high significant increased interincisal distance during follw up.

we concluded that serum relaxin-2 hormone was elevated significantly in patients with TMJ internal deranegement. Also this hormone is detected locally in TMJ joint this may explain its role in the pathogenesis of TMJ internal deranegement.
\end{abstract}

\section{INTRODUCTION}

I nternal derangements of the tempromandibular joint (TMJ) is an abnormal relation of the articular disc to the mandibular condyle and the articular eminence. Jaw pain, clicking of the joint, irregular and limited movement of the jaw are the characteristic symptoms of this disorder ${ }^{(1)}$.

Arthrocentesis of the tempromandibular joint was first described in 1991 by Nitzan et al., (2) , arhrocenthesis with joint lavage was suggested as the simplest form of surgical intervention into the TMJ that is required for treatment of painful limited mouth opening caused by TMJ derangement, besides being the least invasive of all the surgical procedures,arthrocentesis carries a very low risk and it is relatively easy to accomplish as an in office procedure under local anesthesia alone or in combination with conscious sedation, arthrocenthesis is now widely used in various internal derangements as well as diagnostic purposes ${ }^{(3,4,5)}$.Shinya et al., ${ }^{(6)}$ stated that arthrocentesis is a highly efficient procedure to decrease joint pain and increase the range of mouth opening in patients with closed lock of the TMJ,this can be performed under local anesthesia in the outpatient clinic, it is also suggested that lavage under sufficient hydraulic pressure could widen the narrowed joint space release adhesions within the joint space .
Hyaluronic acid (HA) production and quality plays key role in maintaining joint surfaces and lubrication of the synovial membrane in TMJ. HA is a linear polysaccharide consisting of repeating disaccharide units of glucuronic acid and $\mathrm{N}$-acetyl glucosamine linked by B1-3 and B1-4 glycosidic bonds. The clinical effects of HA on pain are probably mediated by several factors. In vitro and in vivo studies indicate that HA can enhance proteoglycan synthesis and prevent its release from the cell matrix ${ }^{(7,8,9) \text {. }}$

The incidence of TMJ disorders is very high in women between the second and fifth decade of life (18-45 years), the prime reproductive age. Women in this age group also have a high incidence of TMJ clicking and tenderness. The female to male ratio in the population is between $3: 1$ to 10:1, again a high predisposition for women of reproductive age.

Relaxin-2 is a hormone found in small amounts in women of reproductive age and in pregnant women. It is not present systemically in men. It has a crucial role in tissue turn over and in childbirth, where it helps relax the pubic symphysis, hence the name relaxin. The pubic symphysis is a fibrocartilage, that also found in the TMJ, where it is the main component in the disc and articular surfaces. The main components of fibrocartilage are collagens and proteoglycans. If either breaks down, the joint won't function properly. Do female reproductive 
hormones contribute to the degradation of the TMJ fibrocartilage? Is there an association between local and systemic hormone levels and TMJ disease in women? Animal studies have shown that relaxin and estrogen induce certain tissue degrading enzymes matrix metalloproteinase (MMPs) in the TMJ disc. The induction of these enzymes leads to loss of collagen and proteoglycans in the disc and can lead to joint degeneration. Progesterone has been shown to have a protective effect ${ }^{(10)}$

\section{PATINTS AND METHODS}

This study was conducted on a forty ASAI females patients aged between 20-40 years old selected from the outpatients clinic of oral and maxillofacial department faculty of dentistry ,suez canal university .They were divided into a) group I( control group) : formed of 20 healthy patients with normal TMJ. B) group II(patients group) : formed of 20 patients complaint from TMJ internal derangement .Patients included in this study were suffering from unilateral or bilateral internal derangement of TMJ , they complaint from TMJ pain, limited TMJ function and movement. We excluded patients who have previous surgicall treatment and those with limited mouth opening caused by only muscle spasm or muscle pain.

All individual were subjected into a) Pre-operative assessment : 1-Medical and dental history. 2- Assessment to standard pain score. 3Measurment of maximal mouth opening . 4Radiographic examination as a- panoramic X-ray . b- MRI. c- Obliqe lateral view

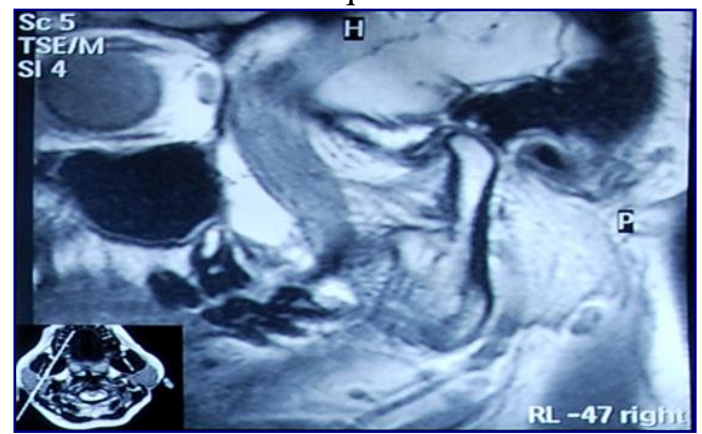

Fig. ( 1 ): Preoperative MRI in closed mouth position, evidence of anterior disc displacement with reduction.

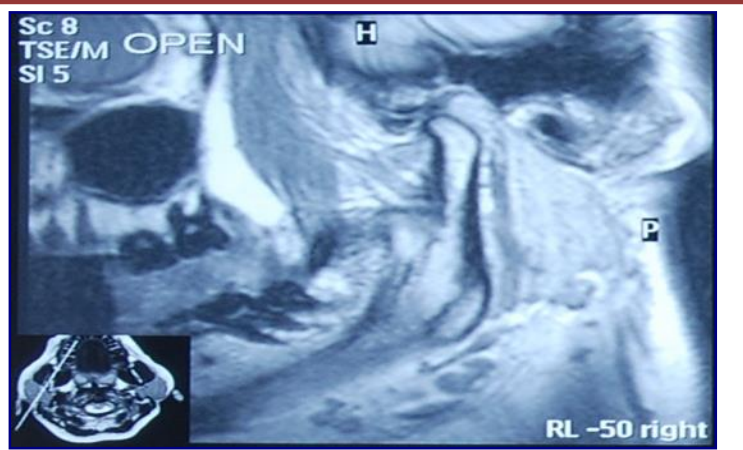

Fig. ( 2 ) : Preoperative MRI in open mouth position, evidence of anterior disc displacement with reduction.

b) Operative procedure : it include 1- Blood samples were drawn from all individual for measurement of relaxin -2 hormone using ELISA. 2- Collection of synovial fluid and arthrocentesis were performed to group II only .The following was done : a- All patients were informed about the procedure and about the materials that used . b- The operative site was prepared aseptically and isolated with sterile draps. c- the skin was penetrated with 20 gauge needle at a point $10 \mathrm{~mm}$ in front of the tragus and $2 \mathrm{~mm}$ below the canthal-tragus line. dAuriculotemporal nerve block was performed with $0.5 \mathrm{ml}$ of the local anesthetic solution .

e- A second needle attached to a syringe introduced $2 \mathrm{~mm}$ anterior to the former one and the upper joint compartment was injected with 2-3 ml of sterile saline and then aspirated again . f- The aspirated synovial fluid was collected on sterile heparin tubes. g- All samples centrifuged at $3000 \mathrm{rpm}$ for 20 minutes to remove the cells before storing the supernatant at $-70 \mathrm{c}$ until assay . h- After termination of procedure one $\mathrm{ml}$ of commercially available sodium hyaluronate injected into upper joint space i- After removal of both needle, the mandible was gently manipulated in order to evaluate joint movement. Finally, the patients practiced opening the mouth as wide as they can . They were instructed for active and isometric opening exercise, protrusive and lateral excursive exercise . Anti-inflammatory were prescribed for three days. 


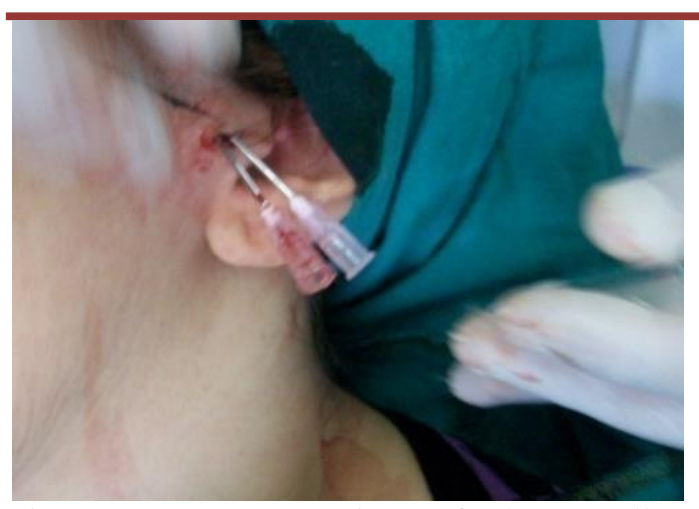

Fig. ( 3 ): Insertion of the needles before arthrocentesis.

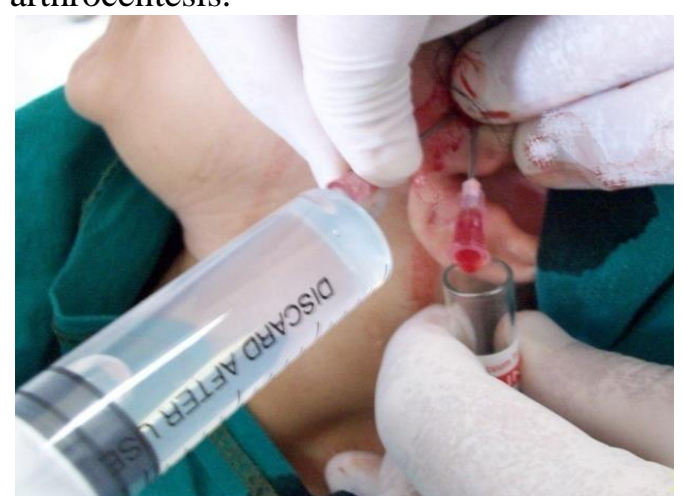

Fig. ( 4 ): Injection of the saline solution into the upper joint compartment during the process of arthrocentesis.

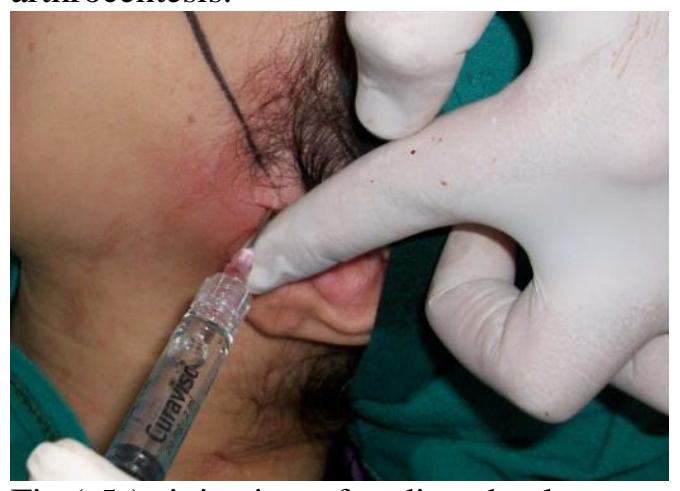

Fig.( 5 ): injection of sodium hyaluronate in upper compartment of the joint which commercially available as ( curavisc $20 \mathrm{mg} / 2 \mathrm{ml}$ syringe) by IDT Biologika GmbH Company,Germany.

C) Post-operative assessment: one week postoperative blood samples were drawn from patients with TMJ internal derangement, to assess relaxin-2 hormone level . Clinical follow -up : it was carried out one week, one month and six months post-operative by : i-Pain scoring through visual analogue scales . ii- Maximal mouth opening through calipers.

Measurement of soluble Relaxin concentration in aspirated synovial fluid and blood withdrawn :
Synovial fluid was sampled from the superior joint cavity by diluted aspiration. The concentration of soluble Relaxin-2 was measured using an immunoenzymometric assay for the quantitative measurement of human Relaxin-2 kit. The measurement of Relaxin-2 was done before and after arthrocentesis which made to the affected joints to detect the relation between Relaxin-2 concentration and the presence of TMJ internal derangement.

\section{RESULTS}

\section{I-Serum relaxin-2 hormone level:}

means serum relaxin -2 hormone level were 9.1+$2.1 \mathrm{pg} / \mathrm{ml}$ in group II, and $4.7+-0.8 \mathrm{pg} / \mathrm{ml}$ in group I . There were significant increase in serum relaxin 2 hormone in patients compared to control $(\mathrm{p}=0.007)($ table 1 ,fig 6)

\begin{tabular}{|l|l|l|l|l|}
\hline & $\begin{array}{l}\text { (group } \\
\text { II) }\end{array}$ & $\begin{array}{l}\text { (group } \\
\text { I) }\end{array}$ & $\mathrm{t}$ & $\mathrm{p}$ \\
\hline $\begin{array}{l}\text { Mean } \\
\pm \text { SD }\end{array}$ & $9.1 \pm 2.1$ & $4.7 \pm 0.8$ & 8.6 & $0.007^{*}$ \\
\hline
\end{tabular}

Table (1): Comparison between group II (patients with TMJ internal derangement) and group I (Control group) in level of Relaxin-2 hormone in serum

*Significant at $\mathrm{P} \leq 0.05$

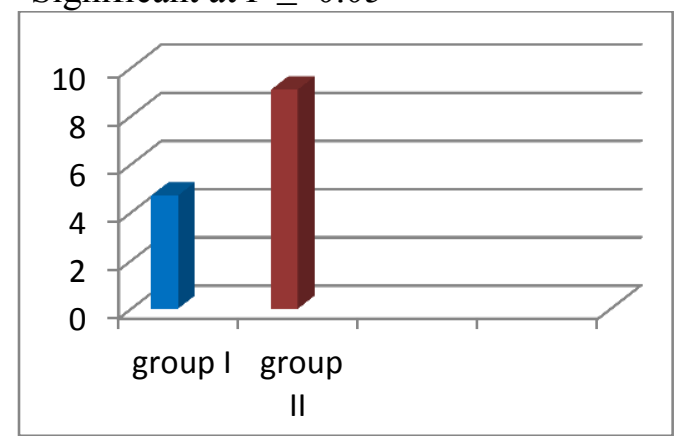

Fig. ( 6 ): the serum relaxin -2 hormone level in patients with TMD (group II ) and(control group)

As regard to serum relaxin -2 hormone after one week post-operative, there were insignificant decrease compared to its pre-operative level in group II $(8.7-+2.5) \mathrm{pg} / \mathrm{ml}$ and $(9.1-+2.1) \mathrm{pg} / \mathrm{ml}$ $(\mathrm{p}=0.55)$

\section{II-synovial fluid relaxin -2 hormone level:}

There was insignificantly decrease in the level of relaxin-2 hormone in the synovial fluid before and after arthrocentesis (3.7-+0.38) pg/ml and (3.5$+0.40) \mathrm{pg} / \mathrm{ml} \mathrm{p}=0.06$ (tab 2, fig 7) 


\begin{tabular}{|l|l|l|l|l|}
\hline & $\begin{array}{l}\text { Before } \\
\text { Mean } \\
\text { SD }\end{array}$ & $\begin{array}{l}\text { After } \\
\text { Mean } \pm \\
\text { SD }\end{array}$ & $\begin{array}{l}\text { Paired } \\
\mathrm{t}\end{array}$ & $P$ \\
\hline $\begin{array}{l}\text { Group } \\
\text { II }\end{array}$ & $3.7 \pm 0.38$ & $3.5 \pm 0.40$ & 1.99 & 0.06 \\
\hline
\end{tabular}

Table ( 2 ) - Synovial fluid relaxin-2 hormone level in patients with anterior disc displacement before and after arthrocentesis in group II .

$\mathrm{P} \geq 0.05$ ( no statistical significant difference )

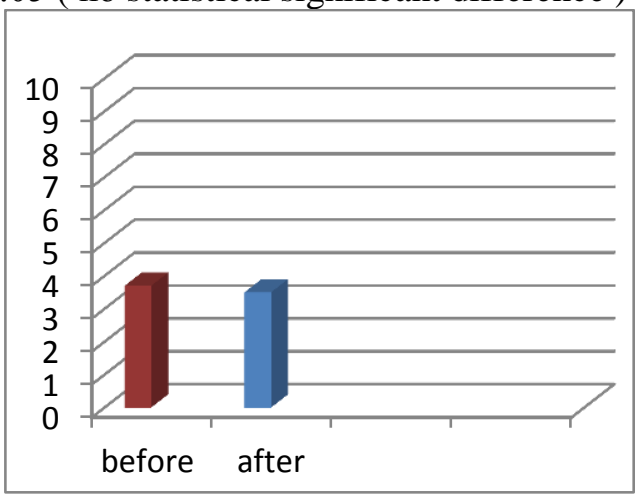

Fig. ( 7 ): level of relaxin-2 hormone in synovial fluid in patients with anterior disc displacement before and after arthrocentesis .

III Clinical evaluation:

There was high significantly decrease in pain score after one week ,one month and at six months postoperative (tab 3,fig 8)

\begin{tabular}{|l|l|l|l|}
\hline & Mean \pm SD & Paired t & $\mathrm{p}$ \\
\hline $\begin{array}{l}\text { Pre- } \\
\text { operative }\end{array}$ & $8.5 \pm 0.9$ & & \\
\hline 1 week & $5.8 \pm 1.2$ & 9.6 & $0.001^{* *}$ \\
\hline 1 month & $3.8 \pm 0.7$ & 24.3 & $0.001^{* *}$ \\
\hline 6 month & $3 \pm 0.6$ & 26.4 & $0.001^{* *}$ \\
\hline
\end{tabular}

Table (3): Pain level before and during follow up after arthrocentesis in groupII.

$(p \leq 0.001)$ There were high significant difference .

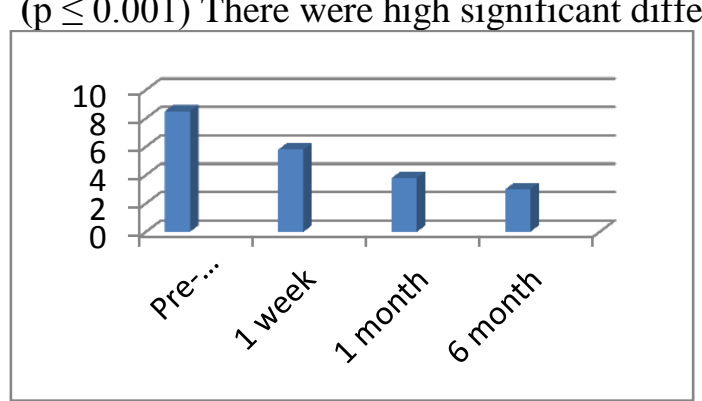

Fig.( 8 ): Pain level before and during follow up after arthrocentesis in groupII.
Also, asregards to mouth opening ,there were a high significant increase in the interincisal distance during follow up (tab 4,fig 9)

\begin{tabular}{|l|l|l|l|}
\hline & Mean \pm SD & Paired t & $\mathrm{p}$ \\
\hline $\begin{array}{l}\text { Pre- } \\
\text { operative }\end{array}$ & $26.4 \pm 6$ & & \\
\hline 1 week & $34 \pm 4.1$ & 16.8 & $0.001^{\text {** }}$ \\
\hline 1 month & $40.8 \pm 2$ & 7.8 & $0.001^{* *}$ \\
\hline 6 month & $45.2 \pm 2.9$ & 7.3 & $0.001^{\text {** }}$ \\
\hline
\end{tabular}

Table (4):- Mouth opening before and during follow up after arthrocentesis in group II . $(\mathrm{p} \leq 0.001)$ There were high significant difference

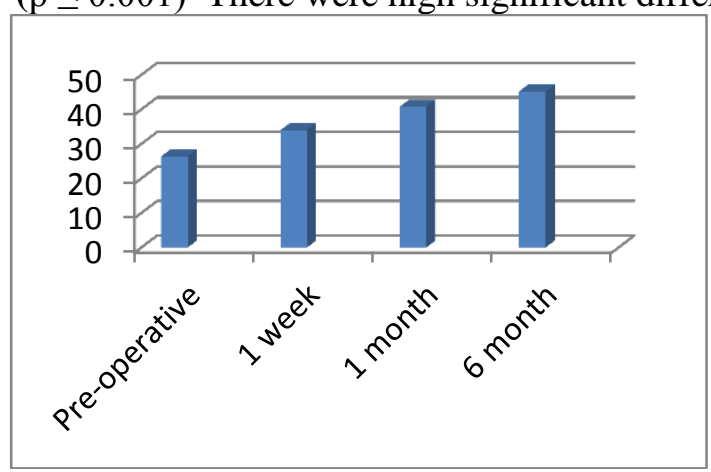

Fig.( 9 ): Mouth opening before and during follow up after arthrocentesis in group II .

\section{DISCUSSION}

Temporomandibular joint disorders (TMJDs) is a term encompassing a spectrum of clinical signs and symptoms which involve the masticatory musculature, the temporomandibular joint (TMJ) and associated structures. While the relative contributions of the muscles of mastication and the joint to TMJD remain unknown. (11)

In a substantial number of patients, these disorders result secondary to TMJ diseases such as osteoarthritis, internal joint derangement and joint hypermobility. ${ }^{(12)}$

Due to poor understanding of the etiopathogenesis of these diseases, and lack of definitive diagnostic or therapeutic approaches, patients often have to tolerate symptoms, including debilitating pain, that substantially impacts on their quality of life over extended periods of time. Identification of specific factors causing or predisposing to TMJ diseases is necessary to enable early recognition and specific therapeutic management of these diseases. ${ }^{(13)}$

In the present study, patients with anterior disc displacement, the serum level of relaxin was significantly increased in comparison to the normal control group. When comparing the level of relaxin2 hormone in patients with TMJDs, before and 
The Role Of Relaxin Hormone In Internal

after arthrocentesis, there were no significant difference .

These results were in agreement with other finding which reported a significant elevation of levels of relaxin in women with TMJD suggesting a potential role of specific sex hormone in causing or predisposing to TMJ degeneration.(14)

In 2012 many authors demonstrated that female reproductive hormones, relaxin, estrogen, and progesterone, and their receptors contribute to the degeneration of temporomandibular joint (TMJ) fibrocartilage potentially contributing to TMJ disorders (TMJDs). $(15,16)$

Also it was demonstrated that relaxin and estrogen increase and progesterone attenuates the expression of specific matrix metalloproteinase (MMP) tissue degrading enzymes and alter the matrix composition of the TMJ disc fibrocartilage. ${ }^{(17)}$

Also recent evidence suggested that relaxin may be produced locally in an autocrine or paracrine in some tissues including joints . ${ }^{(18-19)}$

The explanation of the role of relaxin in TMJDs is by modulating the induction of several matrix metalloproteinase (MMPs) including collagenase and stromylsin the remodeling activities are not limited to reproductive tissues. Also relaxin increase the expression of procollagenase and concomitantly decreases that tissue inhibitor of metalloproteinase (TIMP) and type I collagen . however, despite the implied relationship between relaxin mediated increases in MMP expression and loss of matrix macro molecules, no study has conclusively demonstrated a link between relaxin's induction of MMPs and tissue degradation . ${ }^{(20)}$

In the present study, when comparing the relaxin-2 hormone level in the synovial fluid of TMJ before and after arthrocentesis a non significant difference was noticed and this may be explained on the basis that relaxin hormone may be synthesized locally in the condylar cartilage into small amount as which occur with estrogen which is also synthesized in the joint in small amount and is responsible for TMJ inernal derangement. ${ }^{(21)}$

Finally, It has been concluded that females patients with TMJ internal derangement had elevated level of serum relaxin-2 hormone than normal females patients and still elevated after one week post operative and also relaxin- 2 hormone detected in the synovial membrane of TMJ of these patients .

\section{CONCLUSIONS}

1- arthrocentesis is a simple, office based, non invasive technique.

2- Serum relaxin-2 hormone is elevated significantly in patients with TMJ internal derangement.

3- Relaxin-2 hormone is present locally in the synovial fluid of patients with TMJ internal derangement.

\section{REFERENCES}

1-Emshoff R, Brandlmaier I, Bertram S, and Rudisch A Risk factors for tempromandibular joint pain in patients with disc displacement without reduction $-\mathrm{a}$ magnetic resonance imaging study. J.Oral Rehab, 2003,30 (3) : 537-543.

2. Nitzan D, Dolwick M, and Martinez G (1991): Tempromandibular joint arthrocentesis: A simplified treatment for severe limited mouth opening, J Oral Maxillofac Surg; 48(2): 1163-1176,.

3. Dimitroulis G, Dolwick M, and Martinez G : Tempromandibular joint arthrocentesis and lavage for the treatment of closed lock: A follow up study. Br J Oral Maxillofac Surg; 1995, 33(1): 2326 ,.

4. Alkan A, and Kilic E : A new approach to arthrocentesis of the tempromandibular joint, Int $\mathbf{J}$ Oral Maxillofac Surg.; 2008, 38(1): 85- 96.

5. Yura S, and Totsuka Y : Relationship between effectiveness of arthrocentesis under sufficient pressure and conditions of the tempromandibular joint, J Oral Maxillofac Surg.;2005 , 63(2): 225228.

6. Shinya Y, Yasunori T, Tetsuya y, and Nobuo I : Can arthrocentesis release intracapsular adhesions ? Arthroscopic findings before and after irrigation under sufficient hydraulic pressure, J Oral Maxillofac Surg., 2003 ,61(11):1253-1256 .

7- Escoda-Francolí J, Vázquez-Delgado E, Gay-Escoda C. Scientific evidence on the usefulness of intraarticular hyaluronic acid injection in the management of temporomandibular dysfunction. Med Oral Pathol J 15 (4) 2010, 644-648.

8- El-Hakim IE, Elyamani AO. Preliminary evaluation of histological changes found in a mechanical arthropatic temporomandibular joint (TMJ) exposed to an intraarticular Hyaluronic acid (HA) injection, in a rat model. J Craniomaxillofac Surg, $2011,12-17$.

9- Moreland LW. Intra-articular hyaluronan (hyaluronic acid) and hylans for the treatment of osteoarthritis, mechanisms of action. Arthritis Res Ther Epub J, 2003 ,5: 54-67.

10- Kapila S: At the PCSO, Annual Session TMJ Disorders: Current and Future Innovations in Diagnostics and Therapeutics, 2009 , 27-28.

11-Lipton JA, ship JA, Larach- Robinson D. Estimated prevalence and distribution of reported orofacial pain in the united states . J Am Dent Assoc 1993 : 124:115-121.

12- McNeil C. craniomandibular disorders guidelines for Evaluation, Diagnosis and management the American academy of craniomandibular disorders. 
Quintessence publishing Inc., Chicago; 1993 . 211226

13. Naqvi T, Duong T, Hashem G. Relaxin's induction of metalloproteinases is associated with the loss of collagen and glycosaminoglycans in synovial joint fibrocartilaginous explants. Arthritis Res. Ther, 2005 , 7: 213-216.

14- Kapila S. Hormone-Receptor-MMP Axis in Degenerative TMJ Diseases, J Dent Res, 2012, 1319

15- Park YN, Ahmad N, Hayami T, Kapila S. Relaxin and Estrogen Modulate Targeted Matrix Loss in TMJ Disc, J Dent Res, 2012, 111-119

16-Kapila S. Does the relaxin, estrogen and matrix metalloproteinase axis contribute to degradation of TMJ fibrocartilage? J Musculoskel Neuron Interact 2003;3 (4) :401-5

17- Pearson SJ, Burgess KE, Onamb GL.Serum relaxin levels affect the in vivo properties of some but not all tendons in normally menstruating young women, J Exp Physiol, 2012, 680-685

18- Bennett RG. Relaxin and Its Role in the Development and Treatment of Fibrosis. Transl Res, 2009,154 (1) :1-6.

19- Ehab A. Serum Relaxin Levels and Importance in Systemic Sclerosis Patients. J of American Science, 2012,253-259.

20- Too CKL, Kong JK, Greenwood FC, BryantGreenwood GD. The effect of oestrogen and relaxin on uterine and cervical enzymes: collagenase, proteoglycanase and,$\beta$ - glycuronidase. Acta Endocrinol, $1986,111: 394-403$.

21- Shibin Y, Xianghui X, Shuhui L. locally synthesized estrogen plays an important role in the development of TMD, medical hypotheses , 2009 , 720-722 